\title{
2 The discovery of synthetic proteolytic peptide
} \\ Rina Nakamura ${ }^{1,2}$, Aya Kojima ${ }^{1}$, Motomi Konishi ${ }^{1}$, Masanari Taniguchi ${ }^{1}$, Yusuke Hatakawa ${ }^{1}$ and \\ Toshifumi Akizawa ${ }^{1,2, *}$ \\ ${ }^{1}$ Laboratory of Clinical Analytical Chemistry, Faculty of Pharmaceutical Sciences, Setsunan University, 45-1 \\ Nagaotoge-cho, Hirakata, Osaka 573-0101, Japan; momizit0510@gmail.com \\ ${ }^{2}$ O-Force Co., Ltd., 2-12-31 Shodai-Nakamati, Hirakata, Osaka 573-1152, Japan; momizit0510@gmail.com \\ * Correspondence: momizit0510@gmail.com; Tel.: +81-80-6132-4938
}

Abstract: After screening nearly 1000 synthetic peptides, a synthetic peptide termed JAL-AK22 (KYEGHWYPEKPYKGSGFRCIHI) derived from the BoxA domain in Tob1 protein was found to activate both unfolded and folded proMMP-7. In addition, JAL-AK22 showed auto-proteolytic activity. Interestingly, the smaller derivative of JAL-AK22 termed JAL-TA9 (YKGSGFRMI) also possessed auto-proteolytic activity and cleaved 2 fragment peptides (MMP18-33 and MMP18-40) derived from the prodomain of proMMP-7 under physiological conditions. These proteolytic activities were inhibited by AEBSF, a serine protease inhibitor. Our results demonstrate that a small synthetic peptide consisting of only 9 amino acids has serine protease-like activity and activates proMMP-7 by cleaving the prodomain region. We thus propose calling small peptides possessing with protease-like activity Catalytides (catalytic peptides). We expect that our findings will stimulate the development of novel Catalytides and related applications.
\end{abstract}

Keywords: Catalytide, serine protease-like peptide, Tob1

\section{Introduction}

We reported the microplate assay method for screening the possible inhibitors of MMPs (MMPIs), which are thought to be critically involved in oncogenesis, using with activated recombinant matrix metalloproteinases (actMMPs) and a fluorescence quenching substrate (3163-v) [1-3]. To prepare activated MMP-7 (actMMP-7) from unfold proMMP-7 (u-proMMP-7) expressed in Escherichia coli, refolding step and proteolytic cleavage of prodomain were necessary. The activity of actMMP-7 was monitored with fluorescence intensity produced from 3163-v; thus, the fluorescence intensity reflects the level of cleavage and thereby u-proMMP-7 activation (Scheme 1a). We thought that the chaperone-like peptide could be screened by the microplate assay method. After screening nearly 1,000 peptides, some peptides were found to activate u-proMMP-7 (Scheme 1b). The most potent peptide is termed JAL-AK22 (KYEGHWYPEKPYKGSGFRCIHI), comprising 22 amino acids derived from the BoxA domain in the Tob1 protein (Figure S1a), a membrane-type tyrosine kinase, was found to activate u-proMMP-7, in contrast to the initial expectation (data not shown). The protein encoded by Tob1 is a member of the Tob/BTG family consisted of BTG1, BTG2/Tis21/PC3, ANA/BTG3, BTG4/PC3B and Tob2. The N-terminus region of Tob/BTG family is a highly conserved homology domain that contains BoxA, B and C. According to recent reports, these proteins display antiproliferative activity in a variety of cell types and are involved in the regulation of tumorigenesis [4-6]. However, the role of the BoxA domain of Tob/BTG family protein is still unclear, and there have been no reports of protease activity. Enzymes are generally macromolecular proteins, acting alone or in large complexes. To the best of our knowledge, protease-like activity has never been reported in very small synthetic peptides. In this study, we describe the identification of a small protease-like peptide termed as Catalytide. 
46

47

48

49

50

51

52

53

54

55

56

57

58

59

60

61

62

63

64

\section{Results}

2.1. Screening of proteolytic peptide, JAL-AK22

2.1.1. Screening of proteolytic peptide

Our first aim of this study was to find the chaperone-like peptide, which could affect the refolding of u-proMMP-7 [7]. The reaction mixture containing u-proMMP-7 and fluorescence substrate (3163-v) was incubated at $37^{\circ} \mathrm{C}$ in assay buffer. The fluorescence intensity was measured with excitation at $320 \mathrm{~nm}$ and emission at $390 \mathrm{~nm}$ (Scheme 1b). After screening nearly 1000 synthetic peptides in our peptide library, the synthetic peptide derived from BoxA region of Tob1 protein termed JAL-AK22 (KYEGHWYPEKPYKGSGFRCIHI) (Figure S1a) was found as the activator of u-proMMP-7 (data not shown). However, it was unclear whether JAL-AK22 affected the refolding (Scheme 1b) or cleavage (Scheme 1c) of the prodomain of proMMP-7. To clarify this question, we next analyzed the effect of JAL-AK22 on the activation of folded proMMP-7 (f-proMMP-7), which was prepared by renaturation of u-proMMP-7 (Scheme 1a). As shown in Figure 1, JAL-AK22 significantly enhanced the activation of f-proMMP-7 but slightly reduced the activity of preactivated MMP-7 (actMMP-7) activated by trypsin (Scheme 1a). The f-proMMP-7 alone showed much slower activation apparently due to spontaneous auto-catalysis. These results indicated that JAL-AK22 could activate f-proMMP-7 by proteolytic cleavage of proMMP-7 rather than refolding of u-proMMP-7 [8]. 
(a)
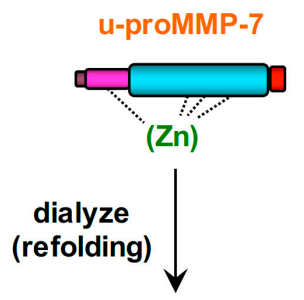

f-proMMP-7

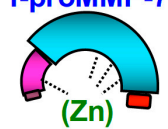

trypsin or
APMA
(cleavage)

actMMP-7

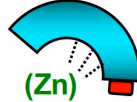

Inhibitor

Substrate

actMMP-7

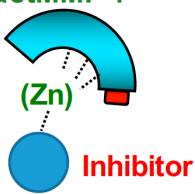

(b)
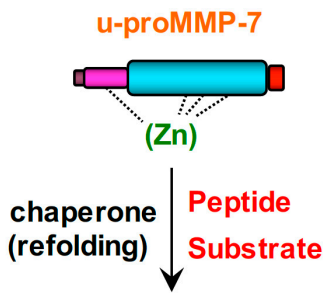

f-proMMP-7
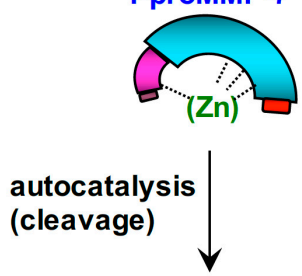

actMMP-7

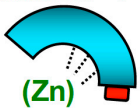

(c)

u-proMMP-7

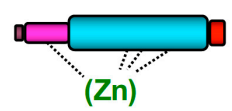

dialyze
(refolding)

f-proMMP-7

(Zn) \begin{tabular}{l|l}
$\begin{array}{l}\text { proteolysis } \\
\text { (cleavage) }\end{array}$ & $\begin{array}{l}\text { Peptide } \\
\text { Substrate }\end{array}$
\end{tabular}

actMMP-7

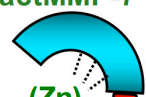

66

Scheme 1. Screening and identification of proteolytic peptides (a) Screening of MMP

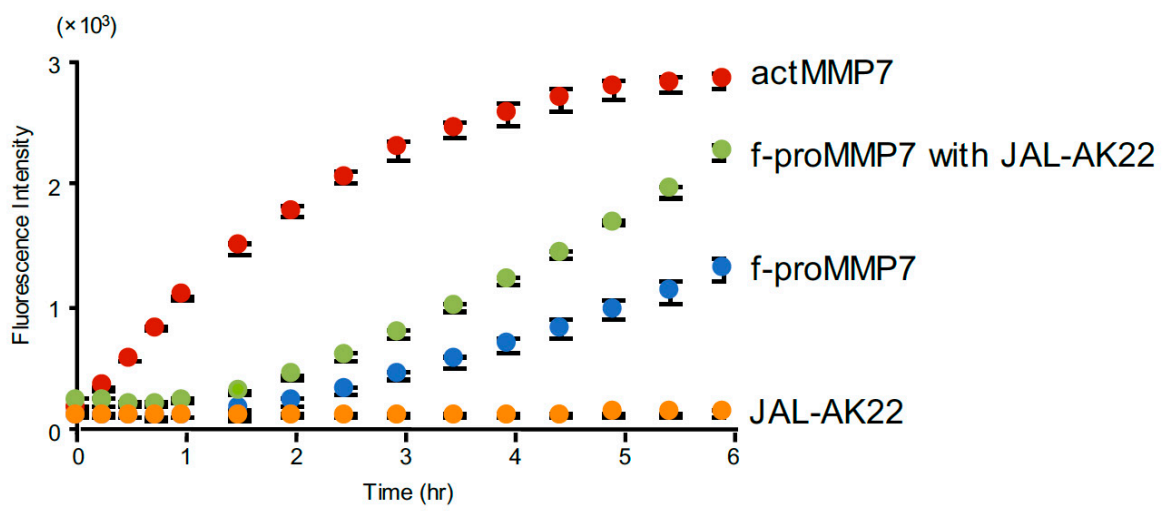


(a)

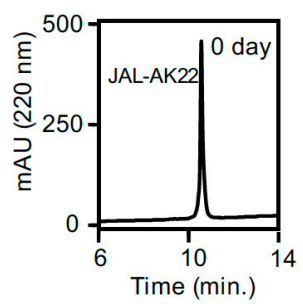

(b)

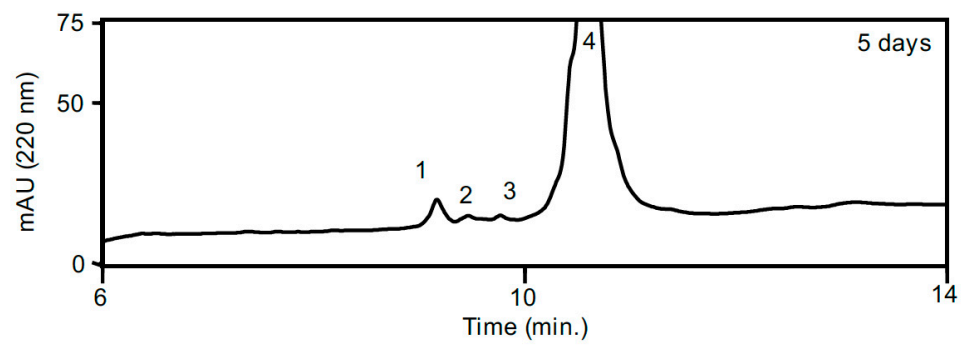

\subsubsection{Auto-proteolytic activity}

In a preliminary analysis of the reaction mixture described above, we noticed a decrease in the JAL-AK22 level after incubation and thus decided to examine its auto-proteolytic activity with or without human serum albumin (HSA). JAL-AK22 was incubated alone at $37^{\circ} \mathrm{C}$ in PBS (pH 7.4), and the incubation mixture was analyzed by HPLC (Figure 2a). The original amount of JAL-AK22 decreased in a time-dependent manner, strongly suggesting that JAL-AK22 has auto-proteolytic activity. The peaks (1-4) in the chromatogram that appeared after 5 days were collected (Figure 2b). By MS analyses, 7 kinds of fragment peptides derived from JAL-AK22 were identified in the reaction mixture (Table 1).
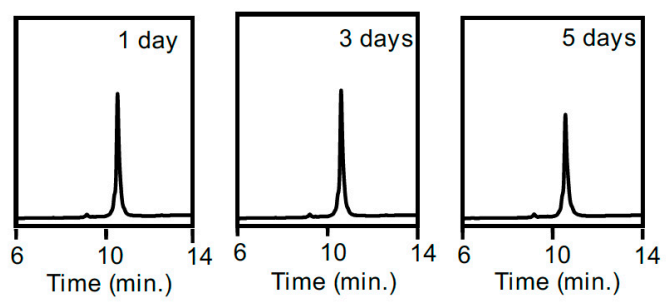

83

$$
\text { Figure 2. Identification of auto-proteolysis activity of JAL-AK22 (a) time dependent }
$$
analysis (b) peak collection

Table 1. Identification of fragment peptides of JAL-AK22

\begin{tabular}{|c|c|c|c|}
\hline Peak & Fragment & Theoretical MS & Experimental MS \\
\hline 1 & KYEGHWYPEKPYK & 1723.84 & 1722.8665 \\
\hline 1 & KYEGHWYPEKPYKGS & 1867.89 & 1866.9345 \\
\hline 2 & KYEGHWYPEKPYKGSGFR & 2228.08 & 2228.1138 \\
\hline 3 & KYEGHWYPEKPY & 1595.74 & 1595.7560 \\
\hline 4 & EKPYKGSGFRC (YKGSGFRCIIHI) I & 2661.35 & 2661.3766 \\
\hline 4 & HWYPEKPYKGSGFRC $(\mathrm{R} \underline{\mathrm{CIH}}) \mathrm{IHI}$ & 2742.35 & 2742.3270 \\
\hline 4 & KYEGHWYPEKPYKGSGFRCIHI (dimmer) & 5386.64 & 5386.6256 \\
\hline
\end{tabular}

On the other hand, 8 kinds of fragment peptides were identified in the reaction mixture including HSA (Figure 3 and Table 2). These data indicated that JAL-AK22 might possess the auto-proteolytic activity. Interestingly, the decreasing ratio of original JAL-AK22 was higher than that of JAL-AK22 alone (Figure S2). It suggests that the auto-proteolytic activity might be enhanced in the presence of HSA. The cleavage sites of JAL-AK22 in this reaction are summarized in Figure 11. 
93 94

(a)
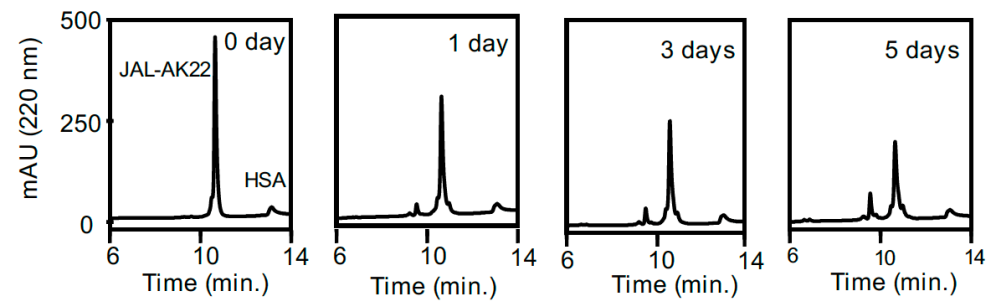

(b)

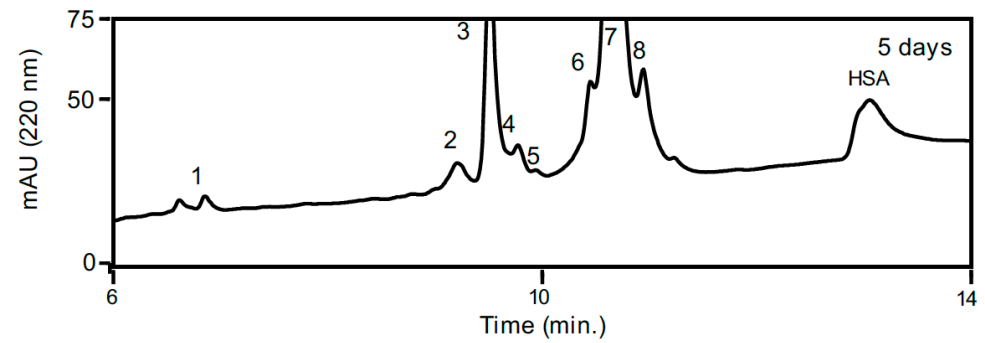

Taken together, we concluded that JAL-AK22 has proteolytic activity and could activate proMMP-7 through proteolytic removal of the prodomain region [6].
Table 2. Identification of fragment peptides of JAL-AK22 with HSA

\begin{tabular}{|c|c|c|c|}
\hline Peak & Fragment & Theoretical MS & Experimental MS \\
\hline 1 & KYEGH & 632.29 & 631.3216 \\
\hline 2 & KYEGHWYPEKPYK & 1723.84 & 1722.9005 \\
\hline 2 & KYEGHWYPEKPYKGS & 1867.89 & 1866.8650 \\
\hline 3 & KYEGHWYPEKPYKGSGFR & 2228.08 & 2228.1402 \\
\hline 4 & KYEGHWYPEKPY & 1595.74 & 1595.7788 \\
\hline 4 & YPEKPYKGSGFRC (SGFR드) I & 2210.05 & 2210.1325 \\
\hline 5 & EKPYKGSGFRC (KPYKGSGFRIIHI) IHI & 3136.64 & 3136.8076 \\
\hline 6 & 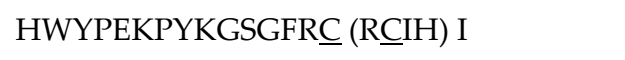 & 2742.35 & 2742.3695 \\
\hline 7 & YEGHWYPEKPYKGSGFR $\underline{C}$ (Rㅡ) I & 2591.20 & 2591.3565 \\
\hline 7 & KYEGHWYPEKPYKGSGFRCIHI (dimmer) & 5386.64 & 5386.7384 \\
\hline 8 & KYEGHWYPEKPYKGSGFRC (CIHI) IHI & 3176.57 & 3176.6497 \\
\hline
\end{tabular}

Figure 3. Identification of auto-proteolysis activity of JAL-AK22 with HSA (a) time dependent analysis (b) peak collection

100 
104

105

106

107

108

109

110

111

112

113

114

115

116

117

118

119

120

121

122

123

124

125

126

127

2.2.1. Auto-proteolytic activity

MS analysis of JAL-AK22 indicated that the Cys residue containing fragment peptides produced by auto-protelytic activity formed the homo/hetero dimers (Table 1 and 2). This reaction is not suitable to identify the proteolytic activity by MS analysis, thus we planed to determine the minimum sequence required for the auto-proteolytic activity. The additional 9 peptides were synthesized and examined the auto-proteolytic activity in the same manner (Figure S2). JAL-TAK22 (KYEGHWYPEKPYKGSGFRMIHI), substituted Cys residue to Met, showed the similar auto-proteolytic activity to JAL-AK22. In addition, JAL-TAY11 (YKGSGFRMIHI) showed more potent than JAL-AK22. The proteolytic activities of the shorter peptides comparison of JAL-TA9 (YKGSGFRMI) were low. As a result, a 9-mer peptide termed JAL-TA9 was found to show the highest auto-proteolytic activity and further investigated for its proteolytic activity (Figure S3b).

The optimal reaction conditions for JAL-TA9 were analyzed by monitoring the auto-proteolytic activity. JAL-TA9 showed potent auto-proteolytic activity between $\mathrm{pH} 7.0$ and 7.5 in the presence of HSA in phosphate buffer (Figure S4a). Judging from the effects of various buffers including PBS, Tris-HCl ( $\mathrm{pH} 7.5$ ), phosphate buffer ( $\mathrm{pH} 7.5)$, assay buffer ( $\mathrm{pH} 7.5)[1,3], \mathrm{PBS}$ was selected for the following experiments (Figure S4b). Among the temperatures tested $\left(4{ }^{\circ} \mathrm{C}\right.$, room temperature, $37^{\circ} \mathrm{C}$ and $70{ }^{\circ} \mathrm{C}$ ), the maximum activity was observed at $37^{\circ} \mathrm{C}$ in $\mathrm{PBS}(\mathrm{pH} 7.4)$ (Figure S4c).

JAL-TA9 (final conc., $0.2 \mathrm{mM}$ ) was incubated alone in PBS at $37{ }^{\circ} \mathrm{C}$, and the reaction mixture was analyzed for 5 days by HPLC. The level of JAL-TA9 was decreased in time dependent manner, along with the formation of several peaks in the HPLC chromatogram (Figure 4a). On day 5, we corrected the 10 peaks (J1 - J10) including JAL-TA9 (J9) (Figure 4b) and identified 8 peptides and 1 amino acid as derivatives from JAL-TA9 (Table 3).

(a)
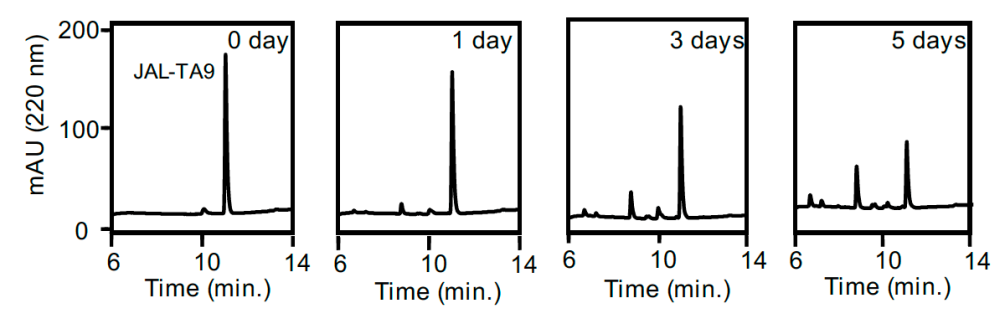

(b)

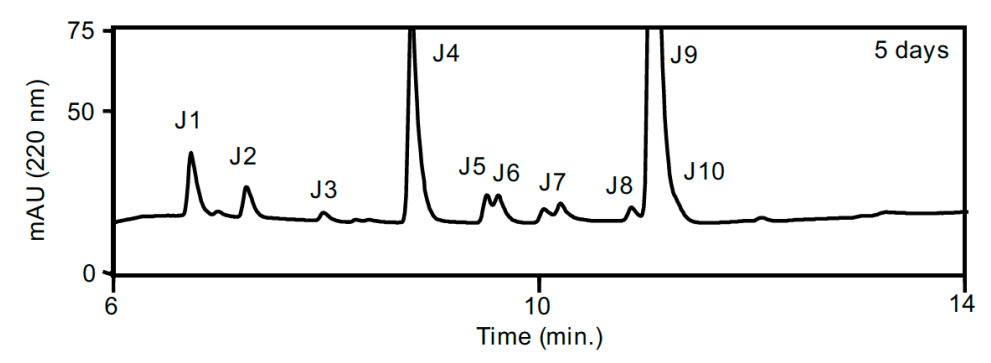

Figure 4. Identification of auto-proteolysis activity of JAL-TA9 (a) time dependent analysis (b) peak collection 
131 Table 3. Identification of fragment peptides of JAL-TA9

132

133

134

135

136

137

138

139

140

141

142

\begin{tabular}{llcc}
\hline Peak & Fragment & Theoretical MS & Experimental MS \\
\hline J1 & YKGSG & 510.24 & 510.2548 \\
J2 & Y & 181.19 & 181.0511 \\
J3 & FR & 321.18 & 321.1800 \\
J4 & YKGSGFR & 813.41 & 813.4260 \\
J5 & YKGSGF & 657.31 & 657.3236 \\
J6 & MI & 262.14 & 262.1452 \\
J7 & YKGSGFRM & 944.45 & 944.6563 \\
J8 & KGSGFRMI & 894.47 & 894.4824 \\
J9 & YKGSGFRMI & 1057.54 & 1057.5705 \\
J10 & FRMI & 565.30 & 565.3011 \\
\hline
\end{tabular}

We next examined the auto-proteolytic activity of JAL-TA9 in the presence of HSA (final conc., $0.025 \% \mathrm{w} / \mathrm{v}$ ). The reaction mixture was analyzed every $1 \mathrm{hr}$ for $6 \mathrm{hrs}$ by HPLC (Figure $5 \mathrm{a}$ ). As expected, similar to JAL-AK22, the auto-catalytic activity of JAL-TA9 was enhanced in presence of HSA (Figure S2b and c). The level of JAL-TA9 was decreased, along with the formation of several peaks in the HPLC chromatogram (Figure 5a). After $1 \mathrm{hr}$ of incubation, the peak corresponding to JAL-TA9 had decreased and almost disappeared after $6 \mathrm{hrs,} \mathrm{with} \mathrm{the} \mathrm{concomitant} \mathrm{detection} \mathrm{of}$ several peaks in the chromatogram (Figure 5b). MS analysis revealed that the new peaks corresponded to new peptides derived from JAL-TA9 (Table 4). The cleavage sites of JAL-TA9 in the presence or absence of HSA are summarized in Figure 11. The specificity for these cleavage sites appeared to be less strict and could not be defined definitively.

(a)
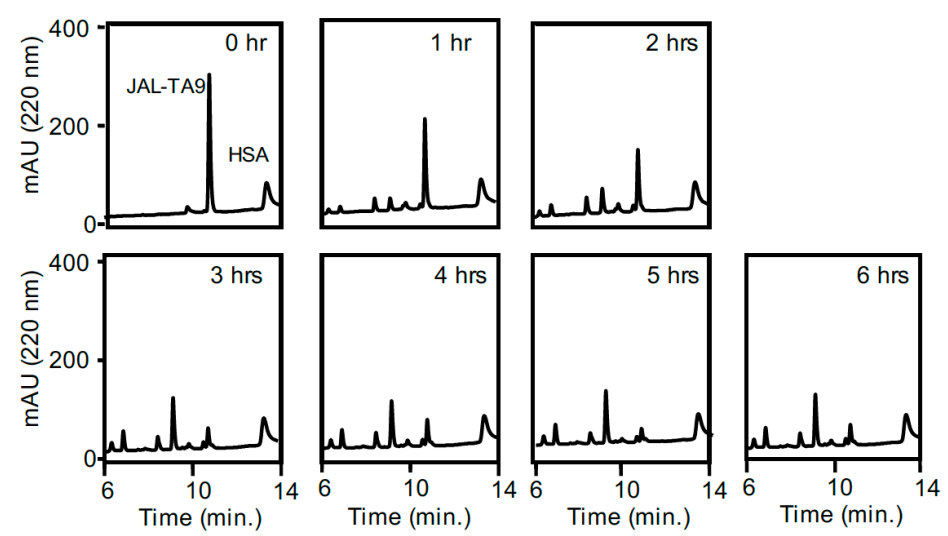

(b)

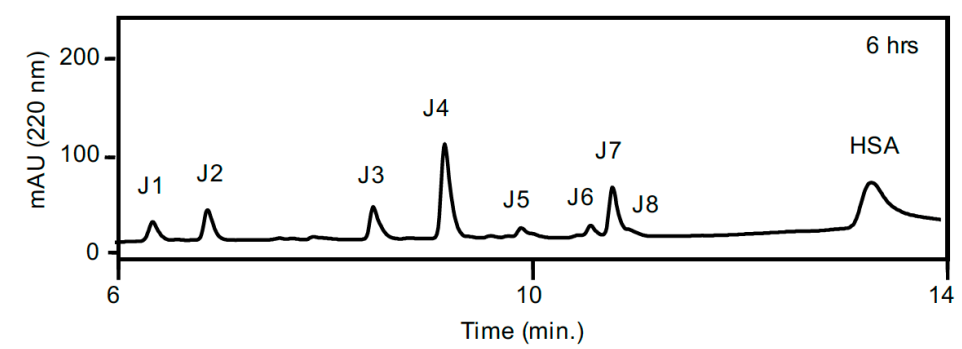


143

144

145

146

\section{7}

148

149

150

151

152

153

154

155

156

157

158

159

160

161

162

Figure 5. Identification of auto-proteolytic activity of JAL-TA9 with HSA (a) time dependent analysis (b) peak collection

Table 4. Identification of fragment peptides of JAL-TA9 with HSA

\begin{tabular}{llcc}
\hline Peak & Fragment & Theoretical MS & Experimental MS \\
\hline J1 & YKGSG & 510.24 & 510.2548 \\
J2 & RMI & 148.24 & 418.2364 \\
J3 & KGSGF & 494.25 & 494.2612 \\
J4 & YKGSGF & 658.32 & 658.3178 \\
J5 & YKGSGFRM & 944.45 & 944.4804 \\
J6 & KGSGFRMI & 894.47 & 894.4902 \\
J7 & YKGSGFRMI & 1057.54 & 1057.5651 \\
J8 & GFRMI & 622.33 & 622.3900 \\
\hline
\end{tabular}

Furthermore, we evaluated the auto-proteolytic activity of JAL-TA9 after filtration of the reaction mixture to deny that these phenomena were due to the contamination of some bacteria or another hydrolytic enzymes. The reaction mixture including HSA was filtered using MILLEX-GV (Millipore, $0.22 \mu \mathrm{m}$ Filter Unit) and TERUMO Syringe (TERUMO, 26G, 1mL) to remove contaminates and then analyzed by the same manner to the reaction mixture without filtration. The chromatograms obtained after incubation for 1 day were the same to those obtained without filtration (Figure S5). These data proved that the auto-proteolytic activity of JAL-TA9 was not due to contamination of bacteria.

In the case of incubation with 4-(2-aminoethyl) benzenesulfonyl fluoride hydrochloride (AEBSF), a serine protease inhibitor, the fragment peptide produced by auto-proteolysis of JAL-TA9 was not appeared on the chromatogram (Figure 6). Peak $C$ in Figure $6 \mathrm{~b}$ was identified as a complex with JAL-TA9 by MS/MS analysis (Figure 7 ). The peaks marked with a star $\left(^{*}\right)$ were not peptides. These data clearly indicated that JAL-TA9 also possessed proteolytic activity and cleaved its own sequence, suggesting that JAL-TA9 can act as a serine protease [9-11]. 
163

(a)

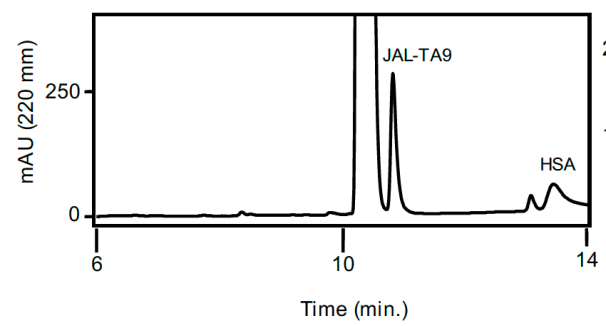

(b)

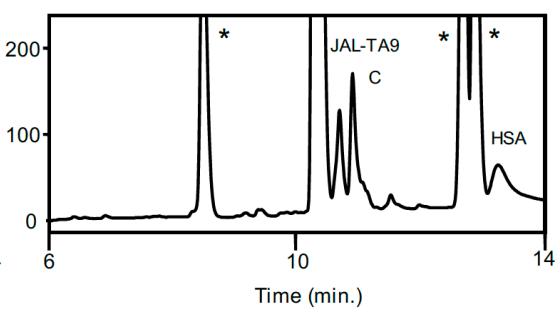

164

165

166

167

168
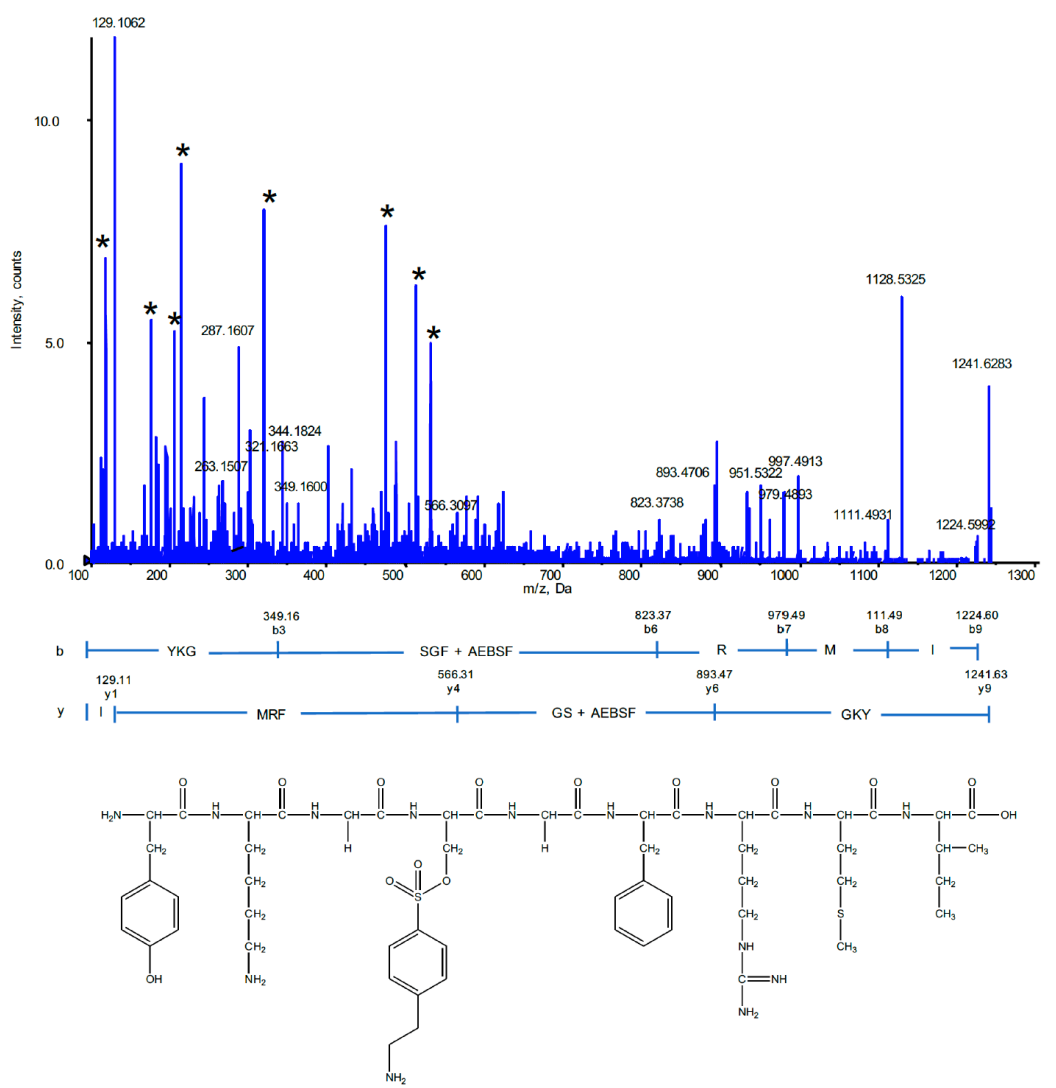
173

174

175

176

177

178

179

180

181

182

183

184

185

2.2.2. Proteolysis of the fragment peptides derived from the prodomain of MMP-7

We next examined the proteolytic activity of JAL-TA9 against the fragment peptides derived from the prodomain of MMP-7, which contained the cleavage site during the activation of proMMP-7 in vivo. Two synthetic fragment peptides derived from the prodomain of MMP-7 (Figure S1b), MMP18-33 (LPLPQEAGGMSELQWE) and MMP18-40 (LPLPQEAGGMSELQWEQAQDYLK), were incubated with JAL-TA9 in the presence of HSA under similar conditions to those described in Figure 3.

Judging from the chromatograms obtained by time dependent analysis, MMP18-33 was stable for 3 days with HSA in PBS (Figure 8a). On the other hand, many peaks were appeared after 1 day incubation in the presence of JAL-TA9 (Figure 8b). To identify these peaks, each peak was collected (Figure 8c) and 6 peaks (M1-6) were identified to the derivatives from MMP18-33 (Table 5). MMP18-40 was also examined according to the same manner, and 7 derivative peptides were identified (Figure 7S). Their cleavage sites were determined (Figure 11).

(a)
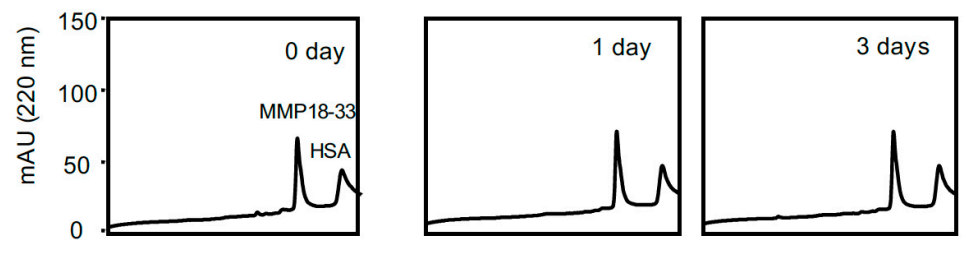

(b)

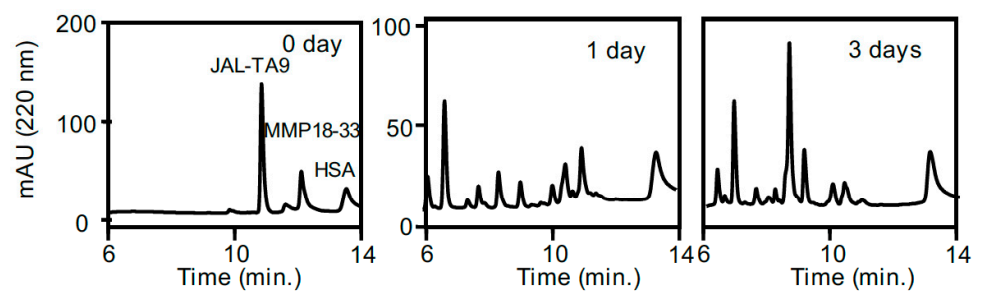

(c)

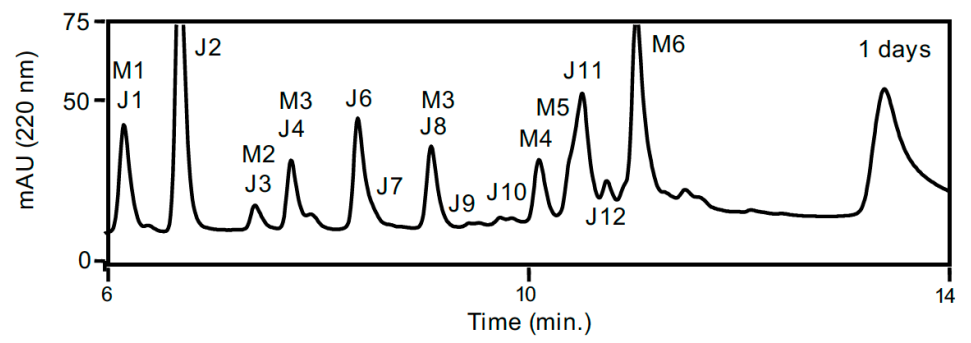

186

187

Figure 8. Proteolytic cleavage of MMP18-33 by JAL-TA9 (a) MMP18-33 alone (b)

188 MMP18-33 with JAL-TA9 (c) peak collection 
Table 5. Identification of fragment peptides of MMP18-33

\begin{tabular}{llcc}
\hline Peak & Fragment & Theoretical MS & Experimental MS \\
\hline M1 & SEL & 347.17 & 347.2215 \\
M2 & EAGGM & 463.17 & 463.2255 \\
M3 & LPQ & 356.21 & 356.3482 \\
M4 & LPLPQ & 566.34 & 566.4081 \\
M5 & SELQWE & 790.35 & 790.4393 \\
M6 & LPLPQEAG & 710.36 & 709.4516 \\
M6 & LPLPQEA & 766.42 & 766.4808 \\
\hline
\end{tabular}

192

We also evaluate the proteolytic activity of JAL-TA9 after filtration of the reaction mixture with HSA as well as the auto-proteolytic activity (Figure 5S). The chromatograms profiles obtained time

198 dependent analysis were the same to those obtained without filtration (Figure 9). These data indicate 199 that the proteolysis of MMP18-33 was not caused by the contamination of some bacteria but caused 200 by JAL-TA9.

(a)
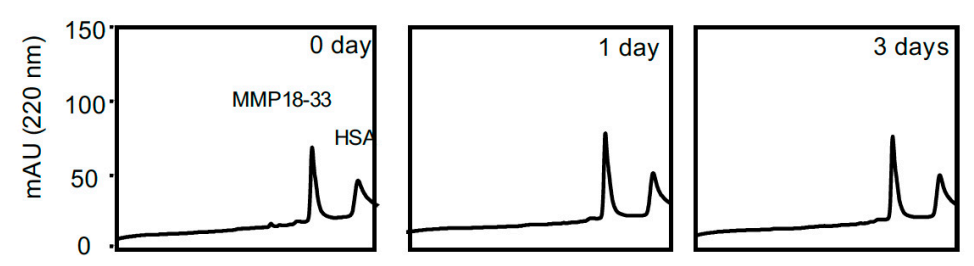

(b)
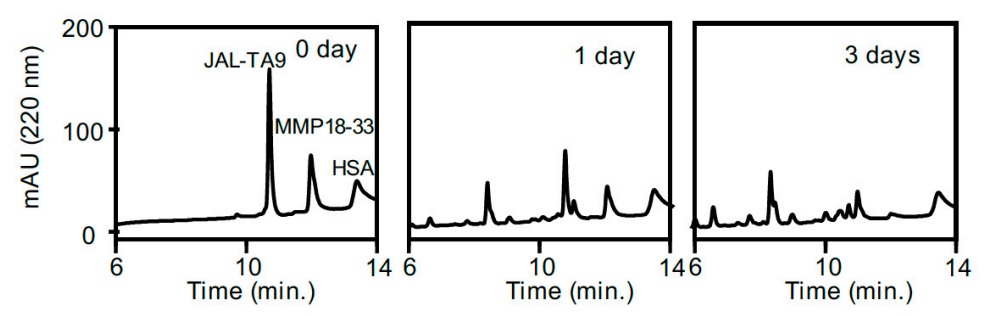

Figure 9. Proteolytic activity of MMP18-33 by JAL-TA9 with filtration (a) MMP18-33 alone (b) MMP18-33 with JAL-TA9

We next examined the inhibitory activity of AEBSF against cleavage reactions (Figure 10). MMP18-33 and MMP18-40 were incubated with JAL-TA9 in the presence of AEBSF, and the reaction mixtures were analyzed by HPLC. On the basis of MS analyses, C1 and C2 in Figure 10b were 
208 identified as the complex of JAL-TA9 and AEBSF, and C3 was identified as the complex of AEBSF 209 and MMP18-40 (Figure S8). The peaks marked with a star $\left(^{*}\right)$ were not peptides and might be the 210 AEBSF products. These data indicated that cleavage reactions of proMMP-7 fragment peptides by 211 JAL-TA9 were inhibited by AEBSF, similar to the auto-proteolysis (Figure 6).

(a)
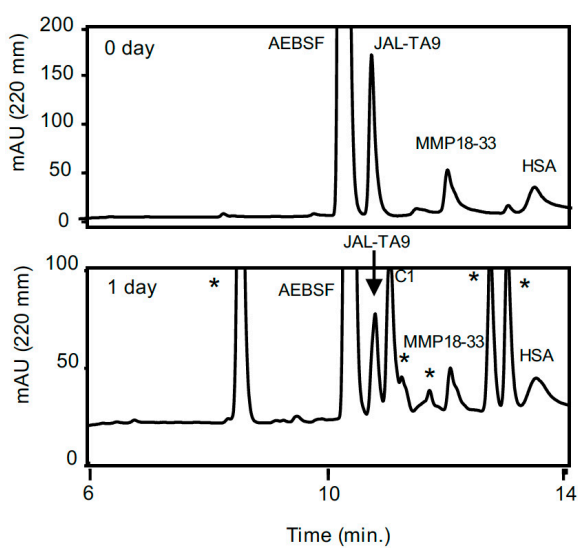

(b)
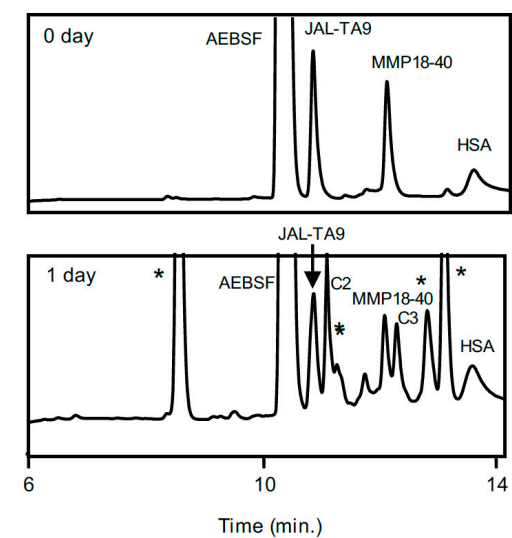

212

213

214

215 Figure 10. Proteolytic cleavage of proMMP-7 fragment peptides by JAL-TA9 in the presence AEBSF (a) MMP18-33 (b) MMP18-40 
As shown in Figure 11, many cleavage sites by auto-proteolysis were identified with or without HSA. In addition, N-terminus regions of both synthetic peptides were cleaved at preferential sites, suggesting some specificity. However, the specificity did not appear to be absolute and thus could not be defined definitively, as in the case of auto-proteolysis.

224

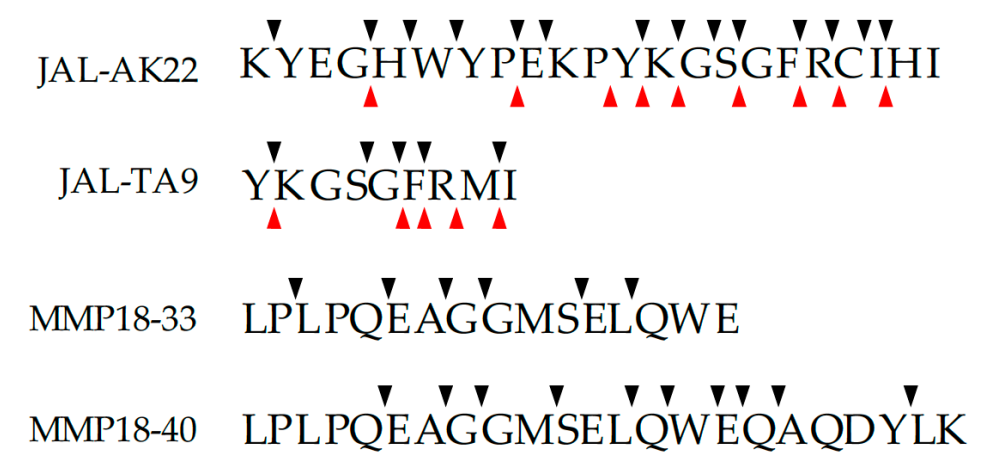

Figure 11. Comparison of cleavage sites in the presence $(\mathbf{V})$ or absence of HSA $(\boldsymbol{\Lambda})$

228

229

230

231

232

The Km and Vmax values of the cleavage reactions of these peptides by JAL-TA9 were $0.17 \mathrm{mM}$ and $0.55 \mathrm{nmol} / \mathrm{hr}$ against MMP18-33 and $0.15 \mathrm{mM}$ and $0.78 \mathrm{nmol} / \mathrm{hr}$ against MMP18-40, respectively (Figure 12 and S8). These values were lower than those of known protein enzymes such as chymotrypsin probably due to the absence of a substrate binding site.

(a)

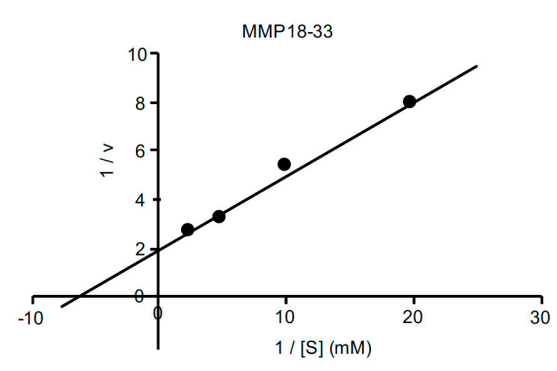

(b)

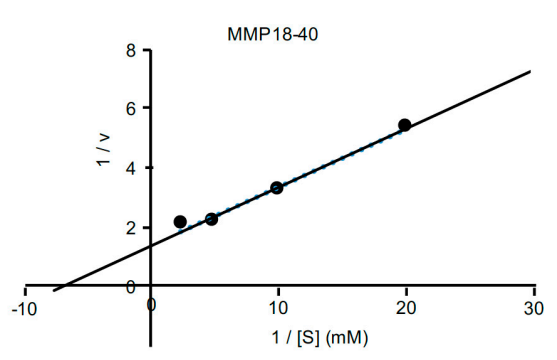




\section{Discussion}

It is the general conception that proteolytic activity is the peculiar to a protein. We also did not have conviction about the existence of proteolytic peptide, therefore we started this project to find a chaperon peptide which could refold the unfolded proMMP-7. In this study, we identified the synthetic peptide possessing the proteolytic activety. It is very exciting and amazing. The high rate of this reaction shows that JAL-TA9 had high activity, potentially higher than that of JAL-AK22, which had not completely degraded even after 5 days of incubation (Figure 3 and 5).

The most important question is an apprehension about the contamination of some bacteria. The proteolytic activity obtained from the reaction mixture after filtration proved that the proteolysis was not caused by the contamination of some bacteria but caused by JAL-TA9 (Figure 5S and Figure 9). In addition, JAL-TA9 showed the proteolytic activity after boiling with hot water or treatment with organic solvents such as $\mathrm{CH}_{3} \mathrm{CN}, \mathrm{CH}_{3} \mathrm{OH}$, DMSO and TFA (data not shown). These data supported the existence of the proteolytic activety of JAL-TA9 itself.

The aim of using HSA at first is to monitor the cleavage reaction against the major protein in blood. We also examined the proteolytic activity of JAL-TA9 against 5 native proteins, $\gamma$-globulin $(\gamma-\mathrm{G})$, rabbit immuno-globulin $\mathrm{G}(\mathrm{IgG})$, cytochrome $\mathrm{C}$ (Cyt-C), lysozyme (Lys) and HSA, by SDS-PAGE with silver staining and HPLC, and none were cleaved by JAL-TA9 (Figure S10). These data suggest that JAL-TA9 may not display serious side effects. Interestingly, HSA enhanced the auto-proteolytic activity of JAL-AK22 and JAL-TA9. However, the effect of HSA is not well understood, but may involve the conformational stabilization of JAL-AK22 and JAL-TA9. AEBSF inhibits the proteolytic activity of JAL-TA9 [9], suggesting the special conformation composed of catalytic triad to show the serine protease-like activity [10,11]. The conformational study by NMR supported the formation of catalytic triad [12]. This conformation may be very flexible and stabilized by the interaction of HSA. The kinetic parameters indicated that the proteolytic activety of JAL-TA9 is weaker than that of protein enzyme such as chymotrypsin due to lack of binding site (Figure 12). In contrast, JAL-TA9 is easy to handle comparison of protein enzyme because of its stability.

Although Tob/BTG proteins are reported as the unstable proteins and difficult to purify probably due to their instability [5], whether any Tob protein itself has proteolytic activity remains unknown. Taken together our finding, it is suggested that Tob/BTG proteins may have proteolytic activity. In addition, our study suggests the possibility that other peptide enzymes derived from degradable and unstable proteins could inform research strategies not only in the field of enzymology but also in molecular biology. The remaining important question is what the substrate of JAL-TA9 is. We now almost finish the investigation of the substrate specificity [13] and structure-activity relationship [14]. 


\section{Materials and Methods}

\subsection{Chemical synthesis of peptides}

Peptides were synthesized from Fmoc-protected L-amino acid derivatives according to the method described by Kojima et al [15]. using an automated peptide synthesizer (model 433A, Applied Biosystems, California, U. S. A., $0.1 \mathrm{mmol}$ scale with preloaded resin). After deprotection according to the manufacturer's protocol, each peptide was purified using reversed-phase HPLC (Capcell Pak C18 column, SG, 10 or 15 mm i.d. x 250 mm; Shiseido Co., Ltd. Japan) with a linear elution gradient from $0.1 \%$ trifluoro acetic acid (TFA) to $50 \%$ or $70 \% \mathrm{CH}_{3} \mathrm{CN}$ containing $0.1 \%$ TFA over $30 \mathrm{~min}$. The flow rate was set at 3.0 or $6.0 \mathrm{~mL} / \mathrm{min}$. The primary peak fractions were collected and then lyophilized. The purity of the synthetic peptides and the progress of the enzymatic reaction were confirmed by analytical reversed-phase HPLC (Capcell Pak C18 column, MGII, 4.6 mm i.d. x $150 \mathrm{~mm}$; Shiseido Co. Ltd., Japan) at a flow rate of $1.0 \mathrm{~mL} / \mathrm{min}$ with a linear elution gradient from $0.1 \%$ TFA to $70 \% \mathrm{CH}_{3} \mathrm{CN}$ containing $0.1 \%$ TFA. The column eluate was monitored with a photodiode-array detector (SPD-M20A; Shimadzu, Japan). Each purified peptide was characterized by ESI-MS using a Qstar Elite Hybrid LC-MS/MS system [15].

\section{2 Preparation of recombinant MMP-7 and identification of proteolytic activity}

The recombinant human proMMP-7 was prepared according to our previous report [1]. Briefly, proMMP-7 was overexpressed in Escherichia coli as a recombinant zymogen (31 kDa), the C-terminus of which bears artificial hexa-histidines. The insoluble fraction of the E. coli extract including u-proMMP-7 was purified in a single step using Ni-NTA resin after solubilization of the precipitates with $8 \mathrm{M}$ urea. The resin-bound recombinant protein was refolded into a f-proMMP-7 by reducing the urea concentration in a stepwise manner. The f-proMMP-7 is activatable by p-amino-phenylmercuric acetate (APMA) or trypsin in an autocatalytic manner. The proteolytic activity of the actMMP-7 was confirmed by a microplate reader assay using the fluorescence quenching substrate, 7-methoxycoumarin-4-yl-acetyl-Pro-Leu-Gly-Leu-[N3-(2,4-dinitrophenyl)-L-2,3-diaminopropionyl]Ala-Arg-NH2 (3163-v: Peptide Institute, Osaka, Japan). Briefly, $60 \mu \mathrm{L}$ of assay buffer for MMP-7 (final conc.: 50 mM Tris-HCl, pH 7.5; 150 mM NaCl; 10 mM CaCl$; 5 \mu \mathrm{M} \mathrm{ZnCl}_{2} ; 0.05 \%$ Brij-35; 3 mM $\left.\mathrm{NaN}_{3}\right)$ containing an appropriate amount of MMP-7 $(30 \mu \mathrm{L})$ and $20 \mu \mathrm{L}$ of a $1 \mathrm{mM}$ peptide solution (final conc., $0.2 \mathrm{mM}$ ) was preincubated in each well of a 96-well microplate at $37^{\circ} \mathrm{C}$ for $15 \mathrm{~min}$, and then $180 \mu \mathrm{L}$ of the fluorescence quenching substrate (3163-v, final conc., $2.5 \mu \mathrm{M})$ was added to initiate the proteolytic reaction. The fluorescence intensity was measured at $320 \mathrm{~nm}$ for excitation and $390 \mathrm{~nm}$ for emission every $15 \mathrm{~min}$ for $2 \mathrm{hrs}$ or every $30 \mathrm{~min}$ for 6 hrs without interrupting the reaction using a fluorescence microplate reader (FP8300, JASCO, Tokyo, Japan). To identify the proteolytic activity of JAL-TA9, u-proMMP-7 or f-proMMP7 was used in place of actMMP-7 (Scheme 1). 


\section{3 Analysis of proteolytic activity and determination of cleavage sites}

JAL-TA9 (final conc., $0.2 \mathrm{mM}$ ) was individually incubated with or without the fragment peptide derived from the prodomain of MMP-7 (final conc., $0.05 \mathrm{mM}$ ) in the presence or absence of human serum albumin (HSA) (final conc., $0.025 \% \mathrm{w} / \mathrm{v}$ ) in PBS (pH 7.4) at $37^{\circ} \mathrm{C}$. A portion of the reaction mixture was analyzed in a time-dependent manner on the analytical HPLC system described above. The peak fractions monitored at $220 \mathrm{~nm}$ were collected into microtubes (Eppendorf Safe-Lock Tubes, $1.5 \mathrm{~mL}$ ).

After lyophilization, the appropriate quantity of $36 \% \mathrm{CH}_{3} \mathrm{CN}$ containing $0.1 \% \mathrm{HCOOH}$ was determined based on the chromatographic peak height and added with stirring by an automatic mixer. The cleavage site were determined by ESI-MS using the flow injection method with $70 \%$ $\mathrm{CH}_{3} \mathrm{CN}$ containing $0.1 \% \mathrm{HCOOH}$ on a Qstar Hybrid LC-MS/MS system (ABI). The flow rate was set at $0.1 \mathrm{~mL} / \mathrm{min}$ [15].

\subsection{Kinetic parameters}

The enzymatic activity of JAL-TA9 was measured using MMP18-33 or MMP18-40 as substrates at 4 different final concentrations (50, 100, 200 and $400 \mu \mathrm{M})$. JAL-TA9 was incubated with MMP18-33 or MMP18-40 in the same manner as in the determination of the proteolytic activity described above. The reaction mixture $(10 \mu \mathrm{L})$ was analyzed on an analytical HPLC at the initial time and after 24 hrs of incubation. The peak heights of MMP18-33 and MMP18-40 gave linear fits on the Lineweaver-Burk plots.

\section{Conclusions}

JAL-TA9 possesses serine protease-like activity [9-11] and activates proMMP-7 by cleaving the prodomain region. To the best of our knowledge, protease-like activity has never been reported in such small synthetic peptides, and we therefore propose that these peptides with protease-like activity, such as JAL-TA9, be called Catalytides (catalytic peptides). We expect that our findings will stimulate the development of novel Catalytides and related applications.

\section{Patents}

T. Yamamoto, T. Akizawa 2016 NOVEL PEPTIDE Patent Pending No US62/275,599, and 2016 NOVEL HYDROLASE-LIKE PEPTIDE AND ITS USE Patent Pending No JP2016-068496

Supplementary Materials: The following are available online at www.mdpi.com/link, Figure S1: Amino acid sequences of Tob1 and MMP-7, S2: Auto-proteolytic activity of JAL-AK22 and JAL-TA9, S3: Structure-activity relationship of JAL-TA9 derivative peptides, S4: Determination of the optimal conditions for the auto-proteolytic activity of JAL-TA9, S5: Auto-proteolytic activity of JAL-TA9 with or without filtration, S6: Cleavage reactions of MMP-7 fragment peptides by JAL-TA9, S7: Identification of cleavage sites, S8: Identification of the complex with JAL-TA9 and AEBSF, S9: Kinetic parameters, S10: Proteolytic activity of JAL-TA9 against native proteins.

Acknowledgments: This work was supported by JSPS KAKENHI Grant Numbers 15K07908. We gratefully thank To Dr. Mitsuaki Yoshida of Cancer Institute and Dr. Hidemitsu Pan-Hou for invaluable discussion and suggestion. 
362

363

364

365

366

367

368

369

370

371

372

373

374

375

376

377

378

379

380

381

382

383

384

385

386

387

388

389

390

391

392

393

394

395

396

397

398

399

400

401

402

403

404

405

406

407

408

409

410

Author Contributions: T.A. were responsible for experimental design and data interpretation. T.A. and R.N. mainly contributed to writing and reversing the manuscript. M.T. was conducted screening of proteolytic peptide. R.N. and A.K. were conducted all experiments, especially HPLC analysis and determination of cleavage sites. R.N., M.K. and Y.H. were contributed to MS analysis. M.K. and R.N. analyzed JAL-TA9 conformation by Computer modeling.

Conflicts of Interest: The authors declare no conflict of interest.

\section{References}

1. Itoh, M.; Masuda, K.; Ito, Y., Akizawa, T.; Yoshioka, M.; Imai, K.; Okada, Y.; Sato, H.; Seiki, M. Purification and Refolding of Recombinant Human proMMP-7 (pro-Matrilysin) Expressed in Escherichia coli and Its Characterization. J. Biochem. 1996, 119, 667-673

2. Oku, N.; Matsukawa, M.; Yamakawa, S.; Asai, T.; Yahara, S.; Hashimoto, F.; Akizawa, T. Inhibitory Effect of Green Tea Polyphenols on Membrane-Type 1 Matrix Metalloproteinase, MT1-MMP. Biol. Pharm. Bull. 2003, 26(9) 1235-1238

3. Taniguchi, M.; Matsumura, K.; Nakamura, R.; Kojima, A.; Konishi, M.; Akizawa, T. MMP-7 cleaves amyloid $\beta$ fragment peptides and copper ion inhibits the degradeation. Biometals. 2017, 30, 797-807, DOI. 10.1007/s10534-017-0048-4

4. Matsuda, S.; Kawamura-Tsuzuku, J.; Ohsugi, M.; Yoshida, M.; Emi, M.; Nakamura, Y.; Onda, M.; Yoshida, Y.; Nishiyama, A.; Yamamoto, T. Tob, a novel protein that interacts with p185erbB2, is associated with anti-proliferative activity. Oncogene 1996, 12, 705-713 DOI. PubMed: 8632892

5. Horiuchi, M.; Takeuchi, K.; Noda, N.; Muroya, N.; Suzuki, T.; Nakamura, T.; Kawamura-Tsuzuku, J.; Takahasi, K.; Yamamoto, T.; Inagaki, F. Structural Basis for the Antiproliferative Activity of the Tob-hCaf1 Complex. JBC. 2009, 284(19), 13244-13255, DOI. 10.1074/jbc.M809250200

6. Winkler, G.S. The Mammalian Anti-Proliferative BTG/Tob Protein Family. J. Cell. Physiol. 2010, 222, 66-72, DOI. 10.1002/jcb.21919.

7. Kojima, A.; Konishi, M.; Noshi, M.; Akizawa, T. Finding of the chaperone peptide. Peptide Science 2008, 2009, 277-280, Proceedings of The 53 ${ }^{\text {th }}$ Japanese Peptide Symposium, The official Journal of Japanese Peptide Society

8. Crabbe, T.; Willenbrock, F.; Eaton, D.; Hynds, P.; Carne, A. F.; Murphy, G.; Docherty, A. J. Biochemical characterization of matrylysin. Activation conformers to the stepwise mechanisms proposed for other matrix metalloproteinases. Biochemistry. 1992, 31, 8500-8507

9. Nakamura, R.; Kojima, A.; Taniguchi, M.; Konishi, M.; Tokumasu, M.; Yamamoto, T.; Akizawa, T. THE NOVEL FINDING OF SERINE PROTEASE-LIKE PEPTIDE. Peptide Science 2016,2017, Proceedings of The $45^{\text {th }}$ Japanese Peptide Symposium, The official Journal of Japanese Peptide Society, Tokto

10. Enrico Di Cera. Serine Proteases. IUBMB Life 2009, 61(5), 510-515, DOI. 10.1002/jub.186

11. Hedstrom, L. Serine Protease Mechanism and Specificity. Chem. Rev. 2002, 102, 4501-4523

12. Nakamura, R.; Konishi, M.; Tanaka, R.; Taniguchi, M.; Kojima, A.; Yamamoto, T.; Akizawa, T. STRUCTURAL ANALYSIS OF THE NOVEL HYDROLASE-LIKE PEPTIDE (JAL-TA9). Peptides 2016. 2016, 111-112, Proceedings of 34th European Peptide Symposium 2016 \& 8th International Peptide Symposium, Leipzig

13. Akizawa, T.; Nakamura, R.; Kojima, A.; Konishi, M.; Taniguchi, M.; Tokumasu, M.; Yamamoto, T. FINDING OF THE NOVEL HYDROLASE-LIKE PEPTIDE (JAL-TA9) DIGESTING A $\beta$ PROTEIN. Peptides 2016. 2016, 28-29, Proceedings of 34th European Peptide Symposium 2016 \& 8th International Peptide Symposium, Leipzig

14. Nakamura, R.; Hatakawa, Y.; Konishi, M.; Taniguchi, M.; Akizawa, T. STRUCTURE-ACTIVITY RELATIONSHIP OF 5-MER PEPTIDES DERIVED FROM JAL-TA9. Peptide Science 2017, 2018, Proceedings of The $53^{\text {th }}$ Japanese Peptide Symposium, The official Journal of Japanese Peptide Society, Kyoto

15. Kojima, A.; Konishi, M.; Akizawa, T. Prion Fragment Peptides Are Digested with Membrane Type Matrix Metalloproteinases and Acquire Enzyme Resistance through $\mathrm{Cu}^{2+}$-binding. Biomolecules 2014, 4, 510-526 (2014); DOI. 10.3390/biom4020510 